\title{
Helmholtz solitons in diffusive Kerr-type media
}

\author{
Julio Sánchez-Curto* and Pedro Chamorro-Posada \\ Departamento de Teoría de la Señal y Comunicaciones e Ingeniería Telemática, Universidad de Valladolid, \\ ETSI Telecomunicación, Paseo Belén 15, 47011 Valladolid, Spain \\ (Received 1 November 2015; published xxxxxx)
}

\begin{abstract}
Soliton evolution at diffusive Kerr-type media is analyzed within the framework of the Helmholtz theory. The angular limitations of previous paraxial studies are overcome when both soliton propagation and diffusion of carriers are allowed to occur along any arbitrary direction. A model including two-dimensional carrier diffusion is proposed and its exact soliton solutions within the weakly nonlocal regime are presented. The restriction of carrier diffusion to a single transverse coordinate leads to the breakdown of the rotational symmetry of the Helmholtz framework and soliton behavior becomes angular dependent. We study the impact of this limitation in an intrinsic angular scenario, such as a nonlinear interface.
\end{abstract}

DOI: 10.1103/PhysRevA.00.003800

\section{INTRODUCTION}

Nonlocal media hosting soliton propagation have enriched the already existing vast field of spatial solitons [1,2]. Thermal media [3] or photorefractive materials [4] have been shown to accommodate thermal [5] or photorefractive [6,7] solitons, respectively. More recently, the reorientational ability of the molecules of a liquid crystal in the nematic phase has been found to induce optical confinement, so that a new type of solitary wave or nematicon can arise [8-10]. Nonlocality hasbeen essential not only in the description of new soliton families, but also in its ability to ease the comprehension of soliton dynamics [11-13] or to overcome certain scenarios that the traditional local models have failed to describe [14-16]. In other cases, the nonlocal response has been essential in the description of higher-order solutions [17,18], soliton interactions [19], and boundary [20,21] and interface [22-24] effects. The works on nematicons at interfaces have been the basis for the design and implementation of nematic crystal valves, which have been proposed as candidates for optical switching devices [25-27].

Regardless of the nature of nonlocal phenomena, soliton propagation in nonlocal media is usually studied using the nonlinear Schrödinger (NLS) equation where the slowly varying envelope approximation (SVEA) is assumed. The scalar nature of the NLS equation has restricted the study of soliton propagation in nonlocal media to broad beams (in relation to its wavelength) that propagate at vanishingly small angles (in relation to the evolution axis). The first type of limitation is overcome when one performs a full vectorial analysis based on Maxwell equations [28,29] that accounts for the small soliton width in relation to its wavelength. This approach has been applied to the study of anisotropic dielectrics where the nonparaxial framework alone has revealed substantial changes in relation to their paraxial counterparts [30,31].

The second restriction involved in the NLS equation, however, is essentially an angular limitation which is removed provided the SVEA is not assumed in the two-dimensional (2D) scalar Helmholtz equation [32]. Described by a scalar model, this type of nonparaxiality deals with broad beams

\footnotetext{
*julsan@tel.uva.es
}

propagating at arbitrary angles and it has been developed 54 in the framework of the Helmholtz theory [33,34], where, 55 for local media, essential corrections to previous paraxial 56 studies have been revealed [35-39]. As regards nonlocal 57 media, longitudinal nonlocal effects have been considered in 58 liquid crystals [40] and, more recently, in thermal media [41]. 59 Even within paraxial propagation contexts, the rapid evolution 60 of the refractive index along the propagation direction must 61 be preserved to accurately describe scenarios where soliton 62 breathing arises or when losses are considered [41]. This type 63 of on-axis nonparaxiality is, however, not addressed in our 64 work. We focus, instead, on the off-axis nonparaxiality arising 65 when shape-preserving beams do not propagate strictly parallel 66 to the longitudinal axis, so that a full angular treatment of the 67 problem is desirable $[9,42]$.

We rely again on the Helmholtz theory to study soliton prop- 69 agation in a diffusive Kerr-type medium where the nonlocal 70 response accounts for the diffusion of carriers that takes place 71 when an optical filed propagates in certain Kerr-type media 72 [43]. However, in order to obtain an adequate description 73 of soliton evolution at arbitrary angles, a full 2D model for 74 the diffusion of carriers preserving the rotational invariance 75 of the Helmholtz framework is required. Otherwise, the 76 results are shown to exhibit a dependence on the propagation 77 angle.

This paper is structured as follows. Section II presents the 79 2D model that rules soliton evolution for diffusive Kerr-type 80 media within the limits of the weakly nonlocal regime. In 81 Sec. III, we study the effects of the breakdown of the rotational 82 symmetry inherent to the Helmholtz framework introduced 83 when carrier diffusion is restricted to a single direction. In 84 Sec. IV, the exact soliton solution for the nonlocal Helmholtz 85 model with 2D carrier diffusion is presented and analyzed. 86 We finally consider, in Sec. V, an inherent angular scenario, 87 such as a nonlinear interface. Section VI summarizes the main 88 conclusions of this work.

\section{THE MODEL}

The time-independent complex field envelope $E(x, z)$ of a 91 continuous-wave TE-polarized beam evolves according to a 92 
93 2D Helmholtz equation,

$$
\frac{\partial^{2} E}{\partial z^{2}}+\frac{\partial^{2} E}{\partial x^{2}}+\frac{\omega^{2}}{c^{2}} n^{2} E=0,
$$

94 where $n$ is the refractive index. This can be decomposed into 95 a linear and a nonlinear part as $n^{2}=n_{l}^{2}+2 n_{l} \delta n$, where $\delta n$ is 96 the field-induced refractive index [43] described by a diffusion 97 equation

$$
D^{2} \nabla_{x z}^{2} \delta n-\delta n+\alpha|E|^{2}=0,
$$

98 where $D$ and $\alpha$ are, respectively, the diffusion and Kerr 99 coefficients.

If we consider a forward-propagating beam $E(x, z)=$ $101\left(2 \kappa n_{l} / \alpha\right)^{1 / 2} u(x, z) e^{j k z}$ and employ the normalizations $\zeta=$ $102 z / L_{D}$ and $\xi=2^{1 / 2} x / w_{0}, w_{0}$ being a transverse scale parameter 103 equal to the waist of a reference Gaussian beam of diffraction 104 length $L_{D}=k w_{0}^{2} / 2$, Eqs. (1) and (2) are transformed into

$$
\kappa \frac{\partial^{2} u}{\partial \zeta^{2}}+i \frac{\partial u}{\partial \zeta}+\frac{1}{2} \frac{\partial^{2} u}{\partial \xi^{2}}+\phi u=0
$$

105 and

$$
d_{0}^{2}\left(\frac{\partial^{2} \phi}{\partial \xi^{2}}+2 \kappa \frac{\partial^{2} \phi}{\partial \zeta^{2}}\right)-\phi+|u|^{2}=0,
$$

106 respectively. In this set of transformations $\kappa=1 / k^{2} w_{0}^{2}$ is 107 a nonparaxiality parameter $[32,33], d_{0}$ is the normalized 108 diffusion coefficient $d_{0}^{2}=2 D^{2} / w_{0}^{2}$, and $\phi=\delta n / 2 \kappa n_{l}$ is the 109 normalized field-induced refractive index. Equations (3) and 110 (4) govern soliton evolution in diffusive Kerr-type media 111 within the Helmholtz nonparaxial framework. Since no as112 sumptions have been made in their derivation, they are fully 113 equivalent to their corresponding Helmholtz and diffusion 114 equations, shown in Eqs. (1) and (2), respectively.

115 According to Eq. (4) the diffusion process can thus take 116 place along any arbitrary direction in the $x z$ plane, since no 117 angular limitation is assumed within the Helmholtz theory. 118 This imposes an essential difference in relation to previous 119 paraxial work [44], which, restricted to vanishingly small 120 angles of propagation, considers only diffusion along the 121 transverse coordinate,

$$
d_{0}^{2} \frac{\partial^{2} \phi}{\partial \xi^{2}}-\phi+|u|^{2}=0
$$

122 Equation (4) represents a generalization of Eq. (5) which 123 can be properly used only in those scenarios restricted to very 124 small angles of propagation where $\partial^{2} \phi / \partial \zeta^{2} \rightarrow 0$. The use 125 of Eq. (5) has been, however, recently proposed to describe 126 soliton evolution at the interface separating diffusive Kerr-type 127 media [45]. We show in Sec. III that in this case the rotational ${ }_{128}$ invariance inherent to the Helmholtz framework is broken and 129 the properties of soliton propagation at large angles may be 130 affected.

\section{A. Weakly nonlocal regime}

132 The study of nonlocal media traditionally distinguishes 133 between weakly and strongly nonlocal regimes, depending 134 on the extent of the response function of the nonlocal media 135 in relation to the optical beam width [44]. When the response 136 function is small compared to the beam width, one works within a weakly nonlocal regime which is mathematically ${ }_{137}$ addressed in a paraxial context provided $d_{0}^{2} \ll 1$ [44]. In ${ }_{138}$ our Helmholtz framework, however, the weakly nonlocal 139 approximation deserves further analysis since the angular 140 content of the problem is also involved.

Equation (4) can be rewritten in the Fourier domain as

$$
\Phi\left(k_{\xi}, k_{\zeta}\right)\left(1+d_{0}^{2} k_{\xi}^{2}+2 \kappa d_{0}^{2} k_{\zeta}^{2}\right)=U\left(k_{\xi}, k_{\zeta}\right),
$$

where $k_{\xi}$ and $k_{\zeta}$ are the transverse and wave numbers, re- ${ }_{143}$ spectively, and $H\left(k_{\xi}, k_{\zeta}\right)=\left(1+d_{0}^{2} k_{\xi}^{2}+2 \kappa d_{0}^{2} k_{\zeta}^{2}\right)^{-1}$ is the 2D ${ }_{144}$ Fourier transform of the response function $R(\xi, \zeta) . U\left(k_{\xi}, k_{\zeta}\right) \quad 145$ and $\Phi\left(k_{\xi}, k_{\zeta}\right)$ denote the 2D Fourier transforms of $|u(\xi, \zeta)|^{2}$ and ${ }_{146}$ $\phi(\xi, \zeta)$, respectively. The response function associated with 147 $H\left(k_{\xi}, k_{\zeta}\right)$ is

$$
\begin{aligned}
R(\xi, \zeta) & =\frac{1}{4 \pi^{2}} \int_{-\infty}^{+\infty} \int_{-\infty}^{+\infty} \frac{e^{j \xi k_{\xi}} e^{j \zeta k_{\zeta}} d k_{\xi} d k_{\zeta}}{1+d_{0}^{2} k_{\xi}^{2}+2 \kappa d_{0}^{2} k_{\zeta}^{2}} \\
& =\frac{1}{2 \pi d_{0}^{2} \sqrt{2 \kappa}} K_{0}\left(\frac{1}{d_{0}} \sqrt{\xi^{2}+\frac{\zeta^{2}}{2 \kappa}}\right)
\end{aligned}
$$

for $(\xi, \zeta) \neq(0,0)$ [46]. $K_{0}$ denotes the modified Bessel ${ }_{149}$ function of the second kind and zero order and its decaying 150 rate is ruled by $d_{0}^{-1}$, so that in the local limit $\left(d_{0} \rightarrow 0\right)$ one 151 has $R(\xi, \zeta) \rightarrow \delta(\xi, \zeta)$. When the response function is narrow 152 in relation to the field intensity, the normalized field-induced 153 refractive index $\phi(\xi, \zeta)=R(\xi, \zeta) *|u(\xi, \zeta)|^{2}$ can be calculated 154 based on its 2D Taylor expansion. Since Eq. (7) verifies 155 that [47]

$$
\int_{-\infty}^{+\infty} \int_{-\infty}^{+\infty} R(\xi, \zeta) d \xi d \zeta=1
$$

and

$$
\frac{1}{2} \int_{-\infty}^{+\infty} \int_{-\infty}^{+\infty} \xi^{2} R(\xi, \zeta) d \xi d \zeta=d_{0}^{2},
$$

one obtains

$$
\phi(\xi, \zeta) \approx|u|^{2}+d_{0}^{2} \frac{\partial^{2}|u|^{2}}{\partial \xi^{2}}+2 \kappa d_{0}^{2} \frac{\partial^{2}|u|^{2}}{\partial \zeta^{2}} .
$$

Equation (10) provides an explicit expression for the nor- 159 malized field-induced refractive index in the weakly nonlocal 160 regime, which is the sum of the local Kerr contribution plus a 161 nonlocal term that accounts for the diffusion processes along 162 both transverse and longitudinal coordinates.

The substitution of Eq. (10) into Eq. (3) leads to

$\kappa \frac{\partial^{2} u}{\partial \zeta^{2}}+i \frac{\partial u}{\partial \zeta}+\frac{1}{2} \frac{\partial^{2} u}{\partial \xi^{2}}+u\left(|u|^{2}+d_{0}^{2} \frac{\partial^{2}|u|^{2}}{\partial \xi^{2}}+2 d_{0}^{2} \kappa \frac{\partial^{2}|u|^{2}}{\partial \zeta^{2}}\right)$

$$
=0 \text {, }
$$

which governs the propagation of solitons at wide angles in 165 diffusive Kerr-type media with a weakly nonlocal response. 166 Equation (11) represents a generalization of the equation 167 found under the paraxial approximation [44], where the term 168 $\kappa \partial^{2} / \partial \zeta^{2} \rightarrow 0$ is neglected.

\section{B. Angular restrictions}

Equation (10) also reveals that the Fourier transform of the 171 response function under the weakly nonlocal approximation 172 
173 is $H\left(k_{\xi}, k_{\zeta}\right) \approx 1-d_{0}^{2} k_{\xi}^{2}-2 \kappa d_{0}^{2} k_{\zeta}^{2}$, which allows us to obtain 174 the set of conditions that address the weakly nonlocal regime 175 within the Helmholtz framework $d_{0} k_{\xi} \ll 1$ and $\sqrt{2 \kappa} d_{0} k_{\zeta} \ll$ 1761 . The maximum longitudinal and transverse wave numbers 177 corresponding to the maximum angle of propagation $\theta=90^{\circ}$ 178 are, respectively, $k_{\zeta, \max }=1 / 2 \kappa$ and $k_{\xi, \max }=1 /(2 \kappa)^{1 / 2}$ [33]. 179 In this case, the former conditions are transformed into one, ${ }_{180} d_{0} \ll(2 \kappa)^{1 / 2}$, which establishes the range of parameters where 181 the weakly nonlocal approximation is valid for all angles of 182 propagation. However, this is a very strict condition which 183 can hardly be fulfilled in our scalar Helmholtz framework 184 where $\kappa \ll 1$. In unscaled coordinates, one has $D \ll \lambda /(2 \pi)$ 185 revealing that the extent of the diffusion process should be 186 even much less than the optical wavelength.

${ }_{187}$ Since we work with broad beams, the validity of this 188 approximation will be restricted to those angles whose 189 transverse wave number verifies $k_{\xi} \ll d_{0}^{-1}$. The strength of 190 the diffusion process thus limits the angular range where 191 the weakly nonlocal approximation is valid. This limitation 192 is taken into account throughout this work, so that soliton 193 parameters are chosen to verify at least $k_{\xi}<d_{0}^{-1}$.

\section{III. ONE-DIMENSIONAL DIFFUSION MODEL}

Exact soliton solutions of the NLS equation with 1D 196 carrier diffusion have been presented in [44]. The assumption 197 that carrier diffusion takes place only along the transverse 198 coordinate is adequate within the paraxial propagation regime. 199 This model has been generalized to a Helmholtz equation 200 including the same type of restricted transverse diffusion [45], 201 which reads

$$
\kappa \frac{\partial^{2} u}{\partial \zeta^{2}}+i \frac{\partial u}{\partial \zeta}+\frac{1}{2} \frac{\partial^{2} u}{\partial \xi^{2}}+u|u|^{2}+d_{0}^{2} \frac{\partial^{2}|u|^{2}}{\partial \xi^{2}} u=0 .
$$

202 Compared to Eq. (11), Eq. (12) retains the rapid evolution of 203 the field envelope along the $\zeta$ coordinate but, simultaneously, 204 discards the contribution of the diffusion process along the 205 same coordinate.

206 The general exact soliton solution of Eq. (12) is

$$
\begin{aligned}
\pm(\xi+V z)= & 2 d_{0} \tan ^{-1}\left(2 d_{0} \sigma^{\prime}\right) \\
& +\frac{\sqrt{1+2 \kappa V^{2}}}{\eta_{0}} \tanh ^{-1}\left(\frac{\sigma^{\prime} \sqrt{1+2 \kappa V^{2}}}{\eta_{0}}\right),
\end{aligned}
$$

207 where $\eta_{0}$ is the soliton peak amplitude, $V$ represents the soliton 208 transverse velocity, and $\sigma^{\prime}$ is the normalized soliton intensity 209 [44],

$$
\sigma^{\prime 2}=\frac{\eta_{0}^{2}-\eta^{2}}{1+2 \kappa V^{2}+4 d_{0}^{2} \eta^{2}}
$$

210 The phase has an explicit expression,

$$
\varphi(\xi, \zeta)=-V \xi \frac{\sqrt{1+2 \kappa \eta_{0}^{2}}}{\sqrt{1+2 \kappa V^{2}}}+\frac{\zeta}{2 \kappa}\left(-1 \pm \frac{\sqrt{1+2 \kappa \eta_{0}^{2}}}{\sqrt{1+2 \kappa V^{2}}}\right) .
$$

211 Figure 1 plots the soliton transverse profiles given by 212 Eqs. (13) and (14) for a diffusive Kerr-type medium with $d_{0}=$

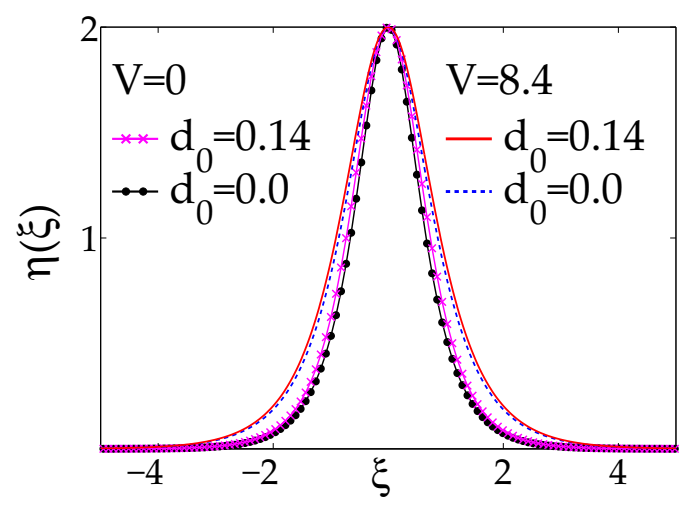

FIG. 1. Soliton transverse profiles for different diffusion coefficients and transverse velocities with 1D carrier diffusion. In all cases, $\eta_{0}=2$ and $\kappa=5 e-3$.

0.14 and a local Kerr medium $\left(d_{0}=0.0\right)$ when two different ${ }_{213}$ transverse velocities are employed. As shown for $V=8.4,214$ where the solid and dotted lines are almost superimposed, the 215 angular correction can even mask the nonlocal contribution. $\quad 216$

This effect can be mathematically captured taking into 217 account the relationship between the transverse velocity and 218 the actual angles of propagation $\theta \tan \theta=(2 \kappa)^{1 / 2} V$ [33], so 219 that Eq. (14) can be rewritten as

$$
\sigma^{\prime}=\cos \theta \sqrt{\frac{\eta_{0}^{2}-\eta^{2}}{1+4 d_{0}^{2} \cos ^{2} \theta \eta^{2}}}=\frac{D_{\theta}}{d_{0}} \sqrt{\frac{\eta_{0}^{2}-\eta^{2}}{1+4 D_{\theta}^{2} \eta^{2}}} .
$$

In Eq. (16) we have defined an angular diffusion coefficient, $\quad 221$

$$
D_{\theta}=d_{0} \cos \theta \text {, }
$$

revealing that the impact of the nonlocal response on soliton 222 evolution depends on the angle of propagation. As the angle 223 of propagation increases nonlocal effects vanish. This can 224 be seen when Eq. (13) is evaluated in the limit $\theta \rightarrow 90^{\circ}$ or, 225 equivalently, $D_{\theta} \rightarrow 0$. While the first term in Eq. (13) becomes 226 negligible,

$$
\lim _{D_{\theta} \rightarrow 0} 2 d_{0} \tan ^{-1}\left(2 D_{\theta} \sqrt{\frac{\eta_{0}^{2}-\eta^{2}}{1+4 D_{\theta}^{2} \eta^{2}}}\right)=0,
$$

the hyperbolic function

$$
\lim _{D_{\theta} \rightarrow 0} \tanh ^{-1}\left(\frac{1}{\eta_{0}} \sqrt{\frac{\eta_{0}^{2}-\eta^{2}}{1+4 D_{\theta}^{2} \eta^{2}}}\right)=\tanh ^{-1}\left(\frac{\sqrt{\eta_{0}^{2}-\eta^{2}}}{\eta_{0}}\right)
$$

remains independent of $d_{0}$, so that one recovers the solution 229 for local Kerr media [33]. As the propagation angle increases 230 and the beam direction departs from the $\zeta$ axis, the transverse 231 nonlocal effects become negligible and local effects prevail. 232 Therefore, soliton behavior becomes angle dependent when 233 carrier diffusion along the $\zeta$ axis is neglected.

We now introduce the exact soliton solutions of the 236 rotationally symmetric Helmholtz equation, (11), including 2D ${ }_{237}$ 


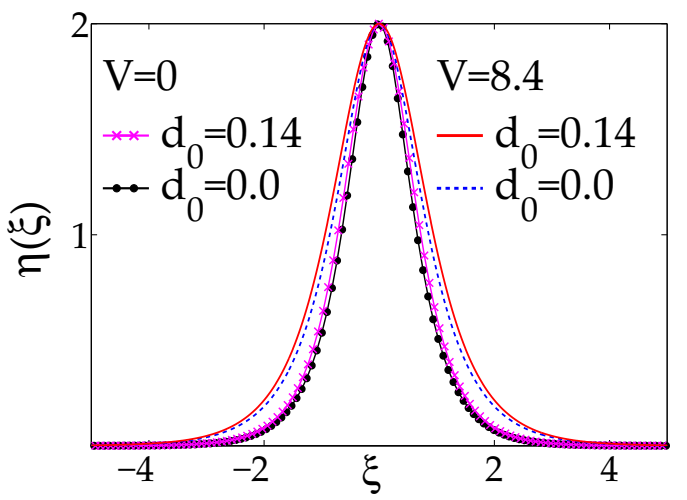

FIG. 2. Soliton transverse profiles for different diffusion coefficients and transverse velocities with 2D carrier diffusion. In all cases, $\eta_{0}=2$ and $\kappa=5 e-3$.

238 carrier diffusion effects and analyze their properties. Using the 239 ansatz $u(\xi, \zeta)=\eta(\xi, \zeta) \exp (i \varphi(\xi, \zeta))$ with the sole assumption 240 that $\varphi$ is a linear combination of both $\xi$ and $\zeta$, the amplitude 241 of the nonlocal Helmholtz soliton $\eta(\xi, \zeta)$ can be expressed in 242 implicit form as

$$
\begin{aligned}
\frac{ \pm(\xi+V z)}{\sqrt{1+2 \kappa V^{2}}}= & 2 d_{0} \tan ^{-1}\left(2 d_{0} \sigma\right) \\
& +\eta_{0}^{-1} \tanh ^{-1}\left(\sigma \eta_{0}^{-1}\right)
\end{aligned}
$$

243 where

$$
\sigma^{2}=\frac{\eta_{0}^{2}-\eta^{2}}{1+4 d_{0}^{2} \eta^{2}}
$$

244 plus a phase term identical to that of Eq. (15).

245 The nonlocal Helmholtz soliton solution preserves the ro246 tational symmetry inherent to the Helmholtz framework. One 247 can easily check that Eqs. (20) and (21) can also be obtained 248 after rotating in the Helmholtz framework the corresponding 249 on-axis solution, $u(\xi, \zeta)=v(\xi) \exp (i \varphi(\zeta))$ [48]. In contrast to 250 Eq. (13), Eq. (20) shows that the nonlocal Helmholtz soliton 251 width preserves its dependency on the nonlocal contribution 252 even when large angles of propagation are involved. This 253 is shown in Fig. 2, where soliton transverse profiles for ${ }_{254} V=8.4$ are no longer superimposed when compared with 255 their corresponding counterparts in Fig. 1.

256 The behavior of the nonlocal Helmholtz soliton is numeri257 cally tested by computing the numerical integration of Eq. (11) 258 using the nonparaxial beam propagation method [49]. The 259 contour plots in Fig. 3 display soliton evolution in diffusive 260 Kerr-type media with $d_{0}=0.2$ (left) and $d_{0}=0.1$ (right) at 261 two transverse velocities.

262 The power flow of the nonlocal Helmholtz soliton is

$$
\begin{aligned}
P_{f} & =\int_{-\infty}^{+\infty}\left(\frac{1}{2 \kappa}+\frac{\partial \varphi(\xi, \zeta)}{\partial \xi}\right)|u(\xi, \zeta)|^{2} d \xi \\
& =\sqrt{1+2 \kappa \eta_{0}^{2}}\left[\eta_{0}+\frac{1+4 d_{0}^{2} \eta_{0}^{2}}{2 d_{0}} \tan ^{-1}\left(2 d_{0} \eta_{0}\right)\right]
\end{aligned}
$$

263 and remains independent of the angle of propagation. Sim264 ulations have been designed to test the validity of Eq. (22). 265 The numerical computation of the integral shown in Eq. (22) (a)

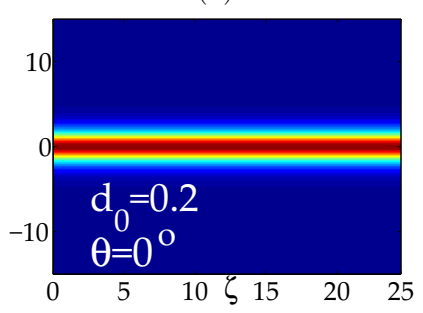

(b)

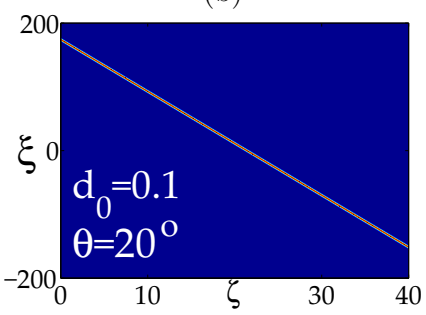

FIG. 3. Nonlocal Helmholtz solitons propagating in diffusive Kerr-type media (a) on axis and (b) off axis, $\theta=20^{\circ}$. In all cases, $\eta_{0}=1$ and $\kappa=1 e-3$.

is plotted with symbols in Fig. 4, while horizontal lines 266 correspond to the analytical result. The agreement between 267 theory and numerics is excellent for all values tested.

This result differs from the one obtained for power flow of 269 the solution given by Eqs. (13)-(15) when the 1D model for 270 carrier diffusion is assumed. In this case, the power flow 271

$$
P_{f}=\sqrt{1+2 \kappa \eta_{0}^{2}}\left[\eta_{0}+\frac{1+4 D_{\theta}^{2} \eta_{0}^{2}}{2 D_{\theta}} \tan ^{-1}\left(2 D_{\theta} \eta_{0}\right)\right]
$$

depends on the angle of propagation through $D_{\theta}$, so that the 272 breakdown of the rotational symmetry manifests again. This 273 is illustrated in Fig. 5(a), where Eq. (23) is displayed as a 274 function of the angle of propagation for different values of $d_{0} 275$ and $\eta_{0}$.

While dotted horizontal lines account for Eq. (22), solid 277 black lines represent the power flow in local Kerr media 278 for $\eta_{0}=1$ and $\eta_{0}=2$ [48]. These two limits constitute, 279 respectively, the maximum and minimum values that Eq. (23) 280 may exhibit. Only for very small angles of propagation $\theta \rightarrow 0 \quad 281$ do the power flows of the 1D and 2D diffusion models 282 agree. As $\theta \rightarrow 90^{\circ}$ and local effects prevail, the power flow 283 tends to the one obtained for local Kerr media. The result 284 of numerical simulations shown in Fig. 5(b) reinforces the 285 angular dependence of the power flow when the 1D model 286 is assumed and reveals the good agreement between theory 287 (lines) and numerics (circles, diamonds, and squares). 288

We now test the validity of the weakly nonlocal regime 289 approximation used so far compared to the complete nonlocal 290 model represented by Eqs. (3) and (4). The field-induced 291

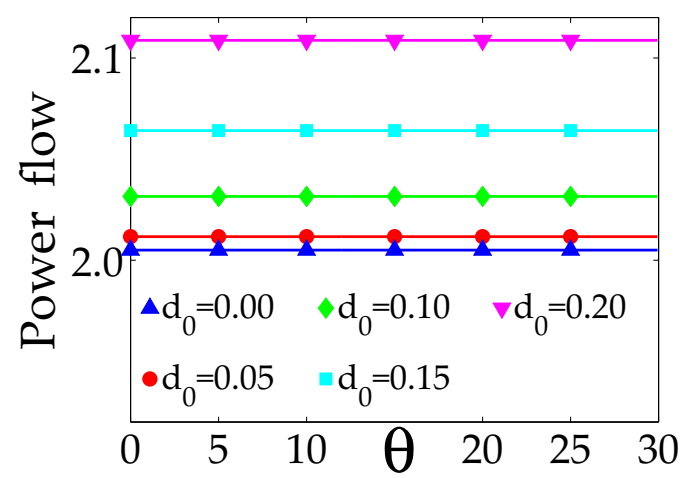

FIG. 4. Soliton power flow for different diffusion coefficients and transverse velocities. In all cases, $\eta_{0}=1$ and $\kappa=2.5 e-3$. 
(a)

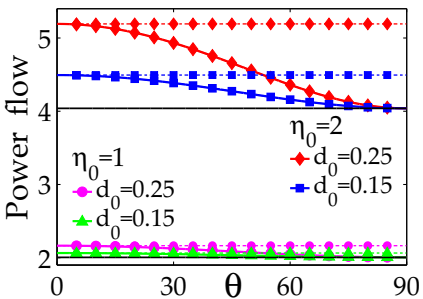

(b)

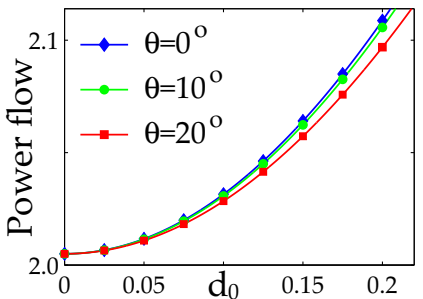

FIG. 5. (a) Power flow dependence on the angle of propagation for different amplitudes and diffusion coefficients with 1D carrier diffusion. (b) Numerical results for the power flow with 1D carrier diffusion. In all cases, $\kappa=2.5 e-3$.

292 refractive index given by Eq. (10) is substituted into Eq. (4) 293 to obtain an estimation of the error assumed upon taking the 294 approximation

$$
\Delta \phi=\max \left\{\left.\left|d_{0}^{2}\left(\frac{\partial^{2} \phi}{\partial \xi^{2}}+2 \kappa \frac{\partial^{2} \phi}{\partial \zeta^{2}}\right)+\right| u\right|^{2}-\phi \mid\right\} .
$$

295 Equation (24) can be rewritten as

$$
\Delta \phi=d_{0}^{4} \max \left\{\left|\frac{\partial^{4}|u|_{\theta=0}^{2}}{\partial \xi^{4}}\right|\right\},
$$

296 where $|u|_{\theta=0}^{2}$ denotes the intensity of the soliton propagating 297 along the longitudinal axis.

\section{NONLINEAR INTERFACES}

In Secs. III and IV, we have shown that the study of 300 diffusive Kerr-type media can be properly addressed only 301 when the diffusion of carriers can occur along any arbitrary 302 direction. Otherwise, the inherent rotational symmetry of зоз the Helmholtz framework is broken and results have been 304 demonstrated to exhibit an angular dependence. It is essential 305 to take this into consideration when one works in nonlinear 306 scenarios that have an inherent angular character, such as 307 nonlinear interfaces [50-53]. Interfaces separating diffusive 308 Kerr-type media have been traditionally studied within the 309 paraxial framework, where modifications to the traditional 310 local models have been reported [54,55]. More recently, the 311 study of such interfaces has been performed in the Helmholtz 312 framework assuming the 1D model described in Sec. III [45]. 313 Nevertheless, this analysis is strictly valid only provided that 314 vanishingly small angles of propagation are involved. The 315 possible impact of this shortcoming is analyzed in the example 316 below.

317 Figure 6 represents soliton evolution at the interface 318 separating local and nonlocal diffusive Kerr-type media with ${ }_{319} d_{0}=0.1$. Solitons, initially propagating in a local medium, 320 impinge a nonlinear interface at an angle of incidence $\theta$, 321 thus undergoing refraction. Soliton evolution in the second 322 medium has been computed twice, depending on the carrier 323 diffusion model assumed. While the $2 \mathrm{D}$ model relies on the 324 numerical integration of Eq. (11), the 1D model is based on 325 Eq. (12).

326 The two pictures in Fig. 6 are visually very similar, although 327 differences can be appreciated under a careful evaluation of 328 the soliton parameters such as the peak amplitude and width.

(a)

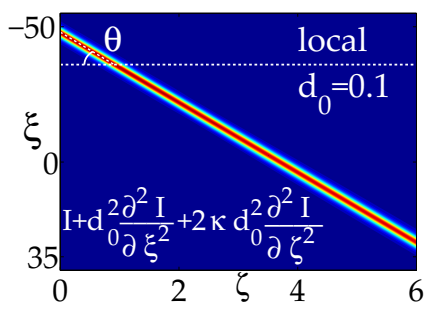

(b)

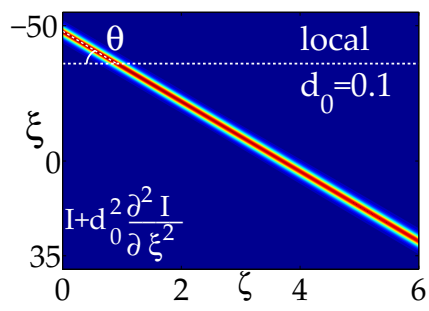

FIG. 6. Soliton evolution at an interface separating local and nonlocal media when (a) the 2D and (b) the 1D diffusion models are used.

Figure 7 represents the evolution of the soliton peak amplitude ${ }_{329}$ for three angles of incidence when the 2D (dotted lines with 330 filled symbols) and 1D (solid lines with open symbols) models 331 are used. Since a sech-type beam is not the exact solution for 332 diffusive Kerr-type media, the beam undergoes breathing upon 333 entering the second medium. For small angles of incidence, 334 such as $10^{\circ}$ or less, differences between the two models 335 are negligible. Dotted and solid lines with circles are almost ${ }_{336}$ superimposed. However, as the angle of incidence increases, 337 the evolution of the soliton peak amplitude displays noticeable 338 differences. The 2D model exhibits a lower peak amplitude, 339 which corresponds to a larger soliton width. The diffusion 340 process is enhanced since carriers can diffuse not only along 341 the transverse, but also along the longitudinal coordinate. 342

Differences between the two models become more ex- 343 plicit at interfaces where, in addition to different diffusion 344 coefficients, local mismatchings, $\Delta=\left(n_{l, 1}^{2}-n_{l, 2}^{2}\right) / n_{l, 1}^{2}$ and 345 $\alpha=\alpha_{2} / \alpha_{1}$, come into play. Such an interface is shown in 346 the two pictures of Fig. 8, which illustrate the evolution of a 347 soliton that initially travels in a nonlocal medium $\left(d_{01}=0.2\right){ }_{348}$ and impinges the interface at an angle $\theta=10.44^{\circ}$. 349

The only difference between the two pictures in Fig. 8 is 350 due to the model assumed for soliton propagation in the first 351 medium, which is 2D in Fig. 8(a) and 1D in Fig. 8(b). The 352 whole field-induced refractive index in the first medium is 353 slightly raised in the 2D case, which makes the critical angle 354 increase. That explains why the soliton propagating at an angle 355

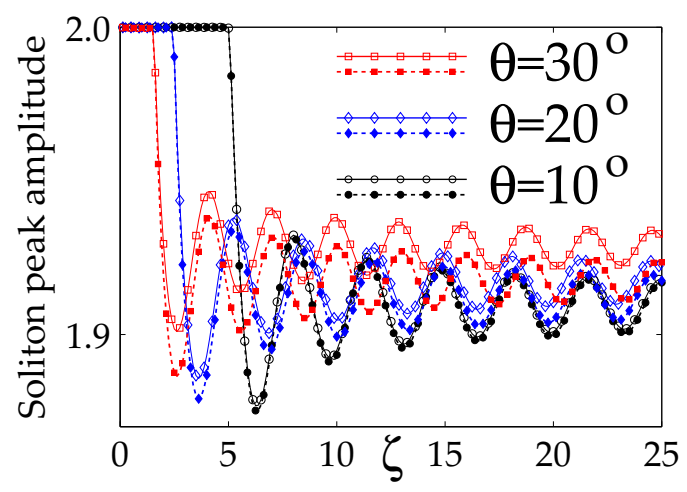

FIG. 7. Evolution of the soliton peak amplitude for an interface between a local and a diffusive Kerr-type medium with $d_{0}=0.1$. In all cases, $\eta_{0}=2$ and $\kappa=1 e-3$. 
(a)
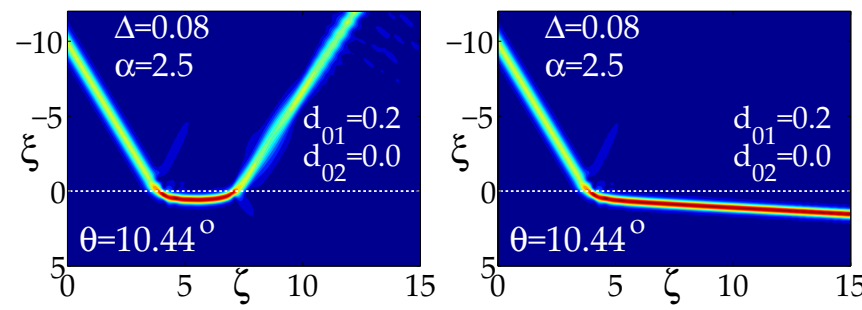

FIG. 8. Soliton evolving at the same interface when (a) a 2D and (b) a 1D propagation model is assumed in the first medium.

356 357 former case but refraction in the latter.

358 Optical switching devices based on soliton reflection and re359 fraction have been proposed for diffusive Kerr-type media [56], 360 photorefractive materials [57,58], and nematic liquid crystals 361 [23,24], where the paraxial approximation has been assumed. 362 Our nonparaxial approach can overcome this limitation and aid 363 the understanding of experiments based on soliton reflection 364 and refraction when nonvanishingly small angles are involved.
The study of this type of nonlocal interfaces is the basis for a 365 forthcoming work on this topic.

\section{CONCLUSIONS}

In this work we have studied the propagation of solitons 368 in diffusive Kerr-type media within the framework of the 369 Helmholtz theory. Our analysis is valid for wide angles of 370 propagation as long as a 2D model for the diffusion of carriers 371 is considered. Exact soliton solutions have been presented 372 and their power flow analysed. A model that only accounts 373 for carrier diffusion along the transverse coordinate breaks 374 the rotational symmetry of the Helmholtz framework, thus 375 restricting its validity to angles of propagation that are not 376 too large. This limitation has been analyzed with an example 377 involving nonlinear interfaces. The validity of our findings 378 has been computationally contrasted with the numerical 379 integration of the corresponding evolution equations, showing 380 an excellent agreement between theory and numerics.

\section{ACKNOWLEDGMENTS}

This work was supported by the Spanish Ministerio de 383 Economía y Competitividad, Project No. TEC2015-69665-R. ${ }_{384}^{384}$
[1] S. Trillo and W. Torruellas, Spatial Solitons (Springer-Verlag, Berlin, 2000).

[2] Z. Chen, M. Segev, and N. Christodoulides, Rep. Prog. Phys. 75, 086401 (2012).

[3] M. D. Iturbe-Castillo, J. J. Sánchez-Mondragón, and S. Stepanov, Opt. Lett. 21, 1622 (1996).

[4] G. C. Duree, Jr., J. L. Shultz, G. J. Salamo, M. Segev, A. Yariv, B. Crosignani, P. Di Porto, E. J. Sharp, and R. R. Neurgaonkar, Phys. Rev. Lett. 71, 533 (1993).

[5] F. Derrien, J. Henninot, M. Warenghem, and G. Abbate, J. Opt. A: Pure Appl. Opt. 2, 332 (2000).

[6] M. Segev, B. Crosignani, A. Yariv, and B. Fischer, Phys. Rev. Lett. 68, 923 (1992).

[7] D.N. Christodoulides and M. I. Carvalho, J. Opt. Soc. Am. B 12, 1628 (1995).

[8] G. Assanto, M. Peccianti, and C. Conti, Opt. Photon. News 14, 44 (2003).

[9] M. Peccianti, C. Conti, G. Assanto, A. De Luca, and C. Umeton, Nature 432, 733 (2004).

[10] M. Peccianti and G. Assanto, Phys. Rep. 516, 147 (2012).

[11] A. W. Snyder and D. J. Mitchell, Science 276, 1538 (1997).

[12] D. J. Mitchell and A. W. Snyder, J. Opt. Soc. Am. B 16, 236 (1999).

[13] C. Conti, M. Peccianti, and G. Assanto, Phys. Rev. Lett. 91, 073901 (2003).

[14] D. Suter and T. Blasberg, Phys. Rev. A 48, 4583 (1993).

[15] O. Bang, W. Krolikowski, J. Wyller, and J. J. Rasmussen, Phys. Rev. E 66, 046619 (2002).

[16] W. Krolikowski, O. Bang, N. I. Nikolov, D. Neshev, J. Wyller, and J. J. Rasmussen, J. Opt. B 6, S288 (2004).

[17] D. Buccoliero, A. S. Desyatnikov, W. Krolikowski, and Y. S. Kivshar, Phys. Rev. Lett. 98, 053901 (2007).
[18] D. Deng and Q. Guo, J. Opt. A: Pure Appl. Opt. 10, 035101 (2008).

[19] M. Peccianti, K. Brzadkiewicz, and G. Assanto, Opt. Lett. 27, 1460 (2002).

[20] B. Alfassi, C. Rotschild, O. Manela, M. Segev, and D. N. Christodoulides, Opt. Lett. 32, 154 (2007).

[21] F. Ye, Y. V. Kartasov, B. Hu, and L. Torner, Opt. Lett. 34, 2658 (2009).

[22] F. Ye, Y. V. Kartashov, and L. Torner, Phys. Rev. A 77, 033829 (2008).

[23] M. Peccianti, A. Dyadyusha, M. Kaczmarek, and G. Assanto, Nat. Phys. 2, 737 (2006).

[24] M. Peccianti, G. Assanto, A. Dyadyusha, and M. Kaczmarek, Phys. Rev. Lett. 98, 113902 (2007).

[25] A. Piccardi, U. Bortolozzo, S. Residori, and G. Assanto, Opt. Lett. 34, 737 (2009).

[26] A. Alberucci, A. Piccardi, U. Bortolozzo, S. Residori, and G. Assanto, Opt. Lett. 35, 390 (2010).

[27] A. Piccardi, A. Alberucci, U. Bortolozzo, S. Residori, and G. Assanto, IEEE Photon. Technol. Lett. 22, 694 (2010).

[28] S. Chi and Q. Guo, Opt. Lett. 20, 1598 (1995).

[29] A. Ciattoni, B. Crosignani, P. Porto, and A. Yariv, J. Opt. Soc. Am. B 22, 1384 (2005).

[30] A. Alberucci and G. Assanto, Opt. Lett. 36, 193 (2011).

[31] A. Alberucci and G. Assanto, Phys. Rev. A 83, 033822 (2011).

[32] G. Fibich, Phys. Rev. Lett. 76, 4356 (1996).

[33] P. Chamorro-Posada, G. S. McDonald, and G. New, J. Mod. Opt. 45, 1111 (1998).

[34] P. Chamorro-Posada, G. S. McDonald, and G. New, J. Opt. Soc. Am. B 19, 1216 (2002).

[35] P. Chamorro-Posada and G. S. McDonald, Phys. Rev. E 74, 036609 (2006). 
[36] J. M. Christian, G. S. McDonald, and P. Chamorro-Posada, Phys. Rev. E 74, 066612 (2006).

[37] J. M. Christian, G. S. McDonald, and P. Chamorro-Posada, Phys. Rev. A 76, 033833 (2007).

[38] J. Sánchez-Curto, P. Chamorro-Posada, and G. S. McDonald, Opt. Lett. 32, 1126 (2007).

[39] J. Sánchez-Curto, P. Chamorro-Posada, and G. S. McDonald, Phys. Rev. A 83, 013828 (2011).

[40] D. W. McLaughlin, D. J. Muraki, M. J. Shelley, and X. Wang, Physica D 88, 55 (1995).

[41] A. Alberucci, C. P. Jisha, N. F. Smyth, and G. Assanto, Phys. Rev. A 91, 013841 (2015).

[42] C. Conti, M. Peccianti, and G. Assanto, Phys. Rev. E 72, 066614 (2005).

[43] E. M. Wright, W. J. Firth, and I. Galbraith, J. Opt. Soc. Am. B 2, 383 (1985).

[44] W. Krolikowski and O. Bang, Phys. Rev. E 63, 016610 (2000).

[45] Z. Shi, Q. Guo, and H. Li, Phys. Rev. A 88, 063848 (2013).

[46] M. Abramowitz and I. A. Stegun, Handbook of Mathematical Functions (Dover, Mineola, NY, 1972).

[47] I. S. Gradshteyn and I. M. Ryzhik, Table of Integrals, Series, and Products (Academic Press, San Diego, CA, 2007).

[48] P. Chamorro-Posada, G. S. McDonald, and G. New, J. Mod. Opt. 47, 1877 (2000).
[49] P. Chamorro-Posada, G. S. McDonald, and G. New, Opt. Commun. 192, 1 (2001).

[50] D. Mihalache, M. Bertolotti, and C. Sibilia, Prog. Opt. 27, 229 (1989).

[51] A. B. Aceves, J. V. Moloney, and A. C. Newell, Phys. Rev. A 39, 1809 (1989).

[52] A. D. Boardman, P. Egan, F. Lederer, and D. Mihalache, in Nonlinear Surface Electromagnetic Phenomena, edited by H. E. Ponath and G. I. Stegeman (North-Holland, Amsterdam, 1991), pp. 73-287.

[53] N. N. Akhmediev, in Nonlinear Surface Electromagnetic Phenomena, edited by H. E. Ponath and G. I. Stegeman (NorthHolland, Amsterdam, 1991), pp. 289-321.

[54] P. Varatharajah, A. B. Aceves, J. V. Moloney, and E. M. Wright, J. Opt. Soc. Am. B 7, 220 (1990).

[55] P. Varatharajah, A. C. Newell, J. V. Moloney, and A. B. Aceves, Phys. Rev. A 42, 1767 (1990).

[56] R. Cuykendall and K. Strobl, J. Opt. Soc. Am. B 6, 877 (1989).

[57] A. D. Boardman, P. Bontemps, W. Ilecki, and A. A. Zharov, J. Mod. Opt. 47, 1941 (2000).

[58] E. Alvarado-Méndez, R. Rojas-Laguna, J. G. Aviña-Cervantes, M. Torres-Cisneros, J. A. Andrade-Lucio, J. C. Pedraza-Ortega, E. A. Kuzin, J. J. Sánchez-Mondragón, and V. Vysloukh, Opt. Commun. 193, 267 (2001). 